\title{
A Spectrally Efficient Transmission Scheme for Signals with Large Bandwidth
}

\author{
Paulo Silva \\ IT/ISE, University of Algarve \\ Campus da Penha, Faro, Portugal \\ Email: psilva@ualg.pt
}

\author{
Rui Dinis \\ IT/FCT, New University of Lisbon \\ Monte da Caparica, Portugal \\ Email: rdinis@fct.unl.pt
}

\begin{abstract}
In this paper we consider single-carrier with frequency-domain equalization (SC-FDE) schemes where the transmission bandwidth is above the symbol rate. To allow high spectral efficiencies, several channels share the same bandwidth. Since the co-channel interference (CCI) levels can be very high, we propose iterative FDE receivers where we jointly detect all users sharing the same channel. Our performance results show that we can have excellent performances, even with several users sharing the same channel. In fact, we can have the maximum theoretical spectral efficiency even with signals that have bandwidth substantially above the symbol rate.
\end{abstract}

\section{INTRODUCTION}

The design of broadband wireless systems presents a considerable challenge since the multipath propagation effects lead to severe time-dispersion effects. Moreover, the systems are required to have good power and spectral efficiencies.

It is widely accepted that single-carrier with frequencydomain equalization (SC-FDE) schemes [1] are the best candidates for the uplink of broadband wireless systems since they can have excellent performance in severely time-dispersive channels and allow low-complexity transmitters [2], [3].

It is well-known that the best spectral efficiency is achieved with a rectangular power spectral density (PSD) with bandwidth equal to the symbol rate. This is equivalent to employ sinc pulses which not just have infinite duration but the relevant part of the pulses span over a large number of symbols. If we also have in mind the high envelope fluctuations and peakto-average power ratio (PAPR), which lead to amplification difficulties, and the synchronization difficulties inherent to the use of sinc pulses, it is not surprising to know that they are not used in practical systems. Current systems employ pulses where the spectrum has a smother shape instead of a "brick wall" shape, typically with a raised-cosine PSD with a given roll-off factor. This means that the actual bandwidth is about $20 \%$ to $50 \%$ above the minimum transmission bandwidth. Since the bandwidth is the scarcest resource in wireless systems, this waste of spectral efficiency is very expensive.

By allowing spectral overlapping we can improve the overall system's spectral efficiency at the expense of some performance degradation due to the resulting adjacent channel

\footnotetext{
${ }^{1}$ This work was partially supported by the FCT-Fundação para a Ciência e Tecnologia (FCT projects PEst-OE/EEI/LA0008/2011, ADCOD PTDC/EEA - TEL/099973/2008 and MPSat PTDC/EEA-TEL/099074/2008.
}

interference (ACI) levels. As an alternative, we can design a receiver able to cope with strong ACI. By taking advantage of the cyclostationary nature of digital signals [4], [5], we can design receivers for channels with strong ACI [6], [7]. This concept can be extended to SC-FDE schemes [8], [9], [10]. A promising receiver for SC-FDE schemes with strong ACI levels is the multiuser frequency-domain receiver proposed in [8] that can be regarded as an iterative block decision feedback equalization (IB-DFE) receiver [11], [12] with multiuser detection and ACI cancellation, allowing excellent performance in the presence of strong ACI levels.

These systems with partial spectral overlapping between adjacent channels can have maximum spectral efficiency regardless of the bandwidth of each channel, provided that the number of channels is large enough, but the receiver complexity can be high.

In this paper we consider a different approach to improve the system's spectral efficiency when the transmission bandwidth of each channel is above the symbol rate. The basic idea is to have several channels sharing the same bandwidth and to perform the joint detection of all channels. Therefore, our receiver can be regarded as the extension of the receiver proposed in [8] to the case where we have co-channel interference (CCI) instead of ACI. However, our scenario is much more difficult because in the ACI case the channels at the edge of the band typically have much lower interference levels than the channels in the middle of the band, while in the CCI case typically all channels can have strong interference levels. For this reason, we also considered turbo receivers where we take advantage of the channel decoder outputs in each iteration of the equalizer/interference canceller.

This paper is organized as follows: Sec. II describes the system model and Sec. III addresses the multiuser frequencydomain receiver for joint equalization and CCI suppression considered in this paper. Sec. IV presents a set of performance results and Sec. V is concerned with the conclusions of the paper.

\section{SySTEM MOdeL}

In this paper we consider the uplink of an SC-FDE system employing cyclic prefix-assisted $(\mathrm{CP})$ block transmission techniques where the transmission bandwidth is above the symbol rate. To allow high spectral efficiencies, we consider 

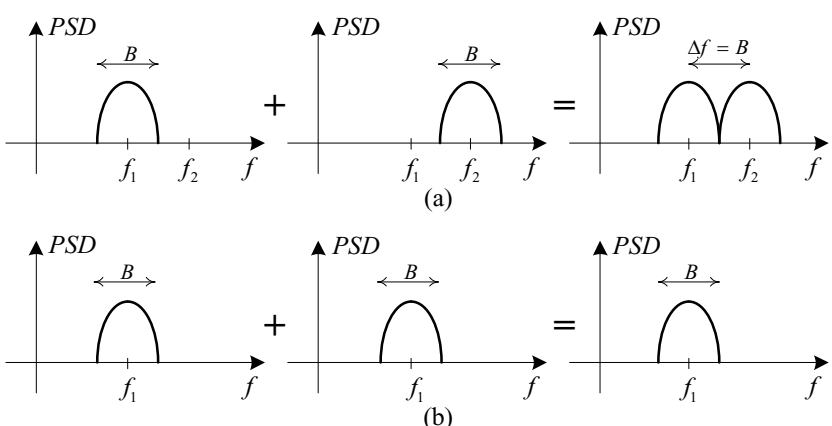

Fig. 1. PSD of the received signals without CCI (a) or with CCI (b).

$P$ users sharing the same bandwidth, as illustrated in Fig. 1. It is assumed that the blocks transmitted by each user have the same dimensions and are synchronized in time (in practice, just a course synchronization is required since some time misalignments can be absorbed by the $\mathrm{CP}$ ).

The time-domain block to be transmitted by the $p$ th user has the form

$$
s_{p}(t)=\sum_{m=-N_{G}}^{M-1} a_{m, p} \xi_{p} h^{(T)}\left(t-m T_{S}\right)
$$

with $T_{S}$ denoting the symbol duration, $N_{G}$ denoting the number of samples at the $\mathrm{CP}, \xi_{p}$ is a suitable scale factor that accounts for the overall attenuation between the $p$ th user and the base station (BS) and $h^{(T)}(t)$ is the adopted pulse shape, which has bandwidth above the symbol rate. The size- $M$ data block to be transmitted is $\left\{a_{m, p} ; m=0,1, \ldots, M-1\right\}$, where $a_{m, p}$ is the $m$ th data symbol, selected from a given constellation under an appropriate mapping rule and $a_{-m, p}=a_{M-m, p}$.

Since the signals associated to each user share the same bandwidth, we need to jointly detect all users. For this propose, the received overall signal is sampled $K$ times each symbol, i.e., the sampling rate is $K / T_{S}$, leading to the time-domain received block $\left\{y_{n} ; n=0,1, \ldots, N-1\right\}$, with $N=K M$. It can be shown that when the CP is longer than the overall channel impulse response for each user the corresponding frequency-domain block is $\left\{Y_{k} ; k=0,1, \ldots, N-1\right\}$, where

$$
Y_{k}=\sum_{p=1}^{P} S_{k, p} H_{k, p}^{(C h)}+N_{k},
$$

with $H_{k, p}^{(C h)}$ denoting the channel frequency response for the $p$ th user and the $k$ th frequency and $N_{k}$ the channel noise for that frequency. The frequency-domain block $\left\{S_{k, p} ; k=\right.$ $0,1, \ldots, N-1\}$ is the discrete Fourier transform (DFT) of $\left\{s_{n, p} ; n=0,1, \ldots, N-1\right\}$. It can be shown that

$$
S_{k, p}=A_{k, p}^{\prime} H_{k}^{(T)},
$$

where the block $\left\{H_{k}^{(T)} ; k=0,1, \ldots, N-1\right\}$ is the DFT of the block $\left\{h_{n}^{(T)} ; n=0,1, \ldots, N-1\right\}$ and

$$
A_{k, p}^{\prime}=\frac{1}{K} A_{k \bmod M, p}
$$

$k=0,1, \ldots, N-1$, with $\left\{A_{k, p} ; k=0,1, \ldots, M-1\right\}$ the DFT of $\left\{a_{m, p} ; m=0,1, \ldots, M-1\right\}$. This means that, apart a constant, the block $\left\{A_{k, p}^{\prime} ; k=0,1, \ldots, N-1\right\}$ is the size- $N$ periodic extension of the DFT of the data block associated to the $p$ th user $\left\{A_{k, p} ; k=0,1, \ldots, M-1\right\}$. This multiplicity in the $A_{k, p}^{\prime}$ is related to the spectral correlations that are inherent to the cyclostationary nature of the transmitted signals [5]. Therefore,

$$
Y_{k}=\sum_{p=1}^{P} A_{k} \bmod M, p H_{k, p}+N_{k},
$$

with

$$
H_{k, p}=\frac{1}{K} \xi_{p} H_{k, p}^{(C h)} H_{k}^{(T)}
$$

denoting the equivalent channel frequency response for the $p$ th user and the $k$ th frequency.

\section{RECEIVER STRUCTURE}

We consider a frequency-domain iterative multiuser receiver based on the receiver proposed in [8]. Each iteration consists of $P$ detection stages, one for each user. It is assumed that the users are ordered in descending order of their power and, when detecting a given user, we cancel the CCI from all users, as well as the residual ISI for the user that is being detected. As with convencional IB-DFE receivers, these interference and residual ISI take into account the reliability of these estimates.

For a given iteration, the detection of the $p$ th user employs the structure depicted in Fig. 2, where we have one length- $N$ frequency-domain feedforward filter, followed by a decimation procedure and $P$ length- $M$ frequency-domain feedback filters (one for each user). The feedforward filter is designed to minimize both the ISI and the CCI that cannot be canceled by the feedback filters, due to decision errors in the previous detection steps. After an inverse DFT (IDFT) operation, the corresponding time-domain outputs are passed through a softdecision device so as to provide an estimate of the data block transmitted by the $p$ th user.

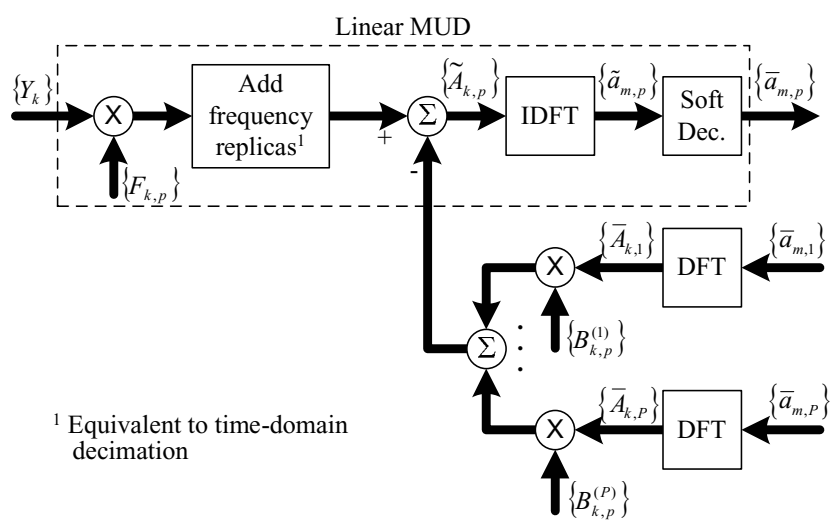

Fig. 2. Detection of the $p$ th user.

For each iteration, the frequency-domain samples associated with the $p$ th user at the detector output are given by

$$
\tilde{A}_{k, p}=\sum_{l=0}^{K-1} F_{k+l M, p} Y_{k+l M}-\sum_{p^{\prime}=1}^{P} B_{k, p}^{\left(p^{\prime}\right)} \bar{A}_{k, p^{\prime}}
$$


where $F_{k, p}(k=0,1, \ldots, N-1)$ and $B_{k, p}^{\left(p^{\prime}\right)}(k=0,1, \ldots, M-$ $1 ; p=1,2, \ldots, P)$ denote the feedforward and the feedback coefficients, respectively. The block $\left\{\bar{A}_{k, p^{\prime}} ; k=0,1, \ldots, M-\right.$ $1\}$ is the DFT of the block $\left\{\bar{a}_{m, p^{\prime}} ; m=0,1, \ldots, M-1\right\}$, where the time-domain samples $\bar{a}_{m, p^{\prime}}, m=0,1, \ldots, M-1$, are the latest average time-domain estimates for the $p^{\prime}$ th user transmitted symbols, i.e., the soft-decisions associated with the block of time-domain samples $\left\{\tilde{a}_{m, p^{\prime}} ; m=0,1, \ldots, M-1\right\}$, the IDFT of $\left\{\tilde{A}_{k, p^{\prime}} ; k=0,1, \ldots, M-1\right\}$. For the $i$ th iteration, $\bar{a}_{m, p^{\prime}}$ is associated with the $i$ th iteration for $p^{\prime}<p$ and with the $(i-1)$ th iteration for $p^{\prime} \geq p$ (in the first iteration, we do not have any information for $p^{\prime} \geq p$ and $\bar{a}_{m, p^{\prime}}=0$ ).

It can be shown that the optimum feedforward coefficients are obtained from a set of $K$ equations:

$$
\begin{aligned}
& \sum_{p^{\prime}=1}^{P}\left(1-\rho_{p^{\prime}}^{2}\right) H_{k+l M, p^{\prime}}^{*} \sum_{l^{\prime}=0}^{K-1} F_{k+l^{\prime} M, p} H_{k+l^{\prime} M, p^{\prime}} \\
& \quad+\alpha F_{k+l M, p}=H_{k+l M, p}^{*}, \quad l=0,1, \ldots, K-1,
\end{aligned}
$$

with

$$
\alpha=\frac{E\left[\left|N_{k}\right|^{2}\right]}{E\left[\left|A_{k, p}\right|^{2}\right]}
$$

and the correlation coefficient $\rho_{p}$ defined as [13]

$$
\rho_{p}=\frac{1}{M} \sum_{m=0}^{M-1} \frac{E\left[a_{m, p}^{*} \hat{a}_{m, p}\right]}{E\left[\left|a_{m, p}\right|^{2}\right]}=\frac{1}{2 M} \sum_{m=0}^{M-1}\left(\rho_{m, p}^{I}+\rho_{m, p}^{Q}\right),
$$

with

$$
\rho_{m, p}^{I}=\frac{E\left[a_{m, p}^{I} \hat{a}_{m, p}^{I}\right]}{E\left[\left|a_{m, p}^{I}\right|^{2}\right]}=\tanh \left(\frac{\left|L_{m, p}^{I}\right|}{2}\right)
$$

and

$$
\rho_{m, p}^{Q}=\frac{E\left[a_{m, p}^{Q} \hat{a}_{m, p}^{Q}\right]}{E\left[\left|a_{m, p}^{Q}\right|^{2}\right]}=\tanh \left(\frac{\left|L_{m, p}^{Q}\right|}{2}\right),
$$

where $L_{m, p}^{I}$ and $L_{m, p}^{Q}$ denote the LLRs (LogLikelihood Ratios) of the "in-phase bit" and the "quadrature bit", associated to $a_{m, p}^{I}$ and $a_{m, p}^{Q}$, respectively, given by ${ }^{2}$

$$
L_{m, p}^{I}=\frac{2}{\sigma_{p}^{2}} \tilde{a}_{m, p}^{I} \text { and } L_{m, p}^{Q}=\frac{2}{\sigma_{p}^{2}} \tilde{a}_{m, p}^{Q},
$$

respectively, where

$$
\sigma_{p}^{2}=\frac{1}{2} E\left[\left|a_{m, p}-\tilde{a}_{m, p}\right|^{2}\right] \approx \frac{1}{2 M} \sum_{m=0}^{M-1} E\left[\left|\hat{a}_{m, p}-\tilde{a}_{m, p}\right|^{2}\right] .
$$

$\rho_{m, p}^{I}$ and $\rho_{m, p}^{Q}$ can be regarded as the reliabilities associated to the "in-phase" and "quadrature" bits of the $m$ th symbol of the $p$ th user (naturally, $0 \leq \rho_{m, p}^{I} \leq 1$ and $0 \leq \rho_{m, p}^{Q} \leq 1$ ) (for the first iteration, $\rho_{m, p}^{I}=\rho_{m, p}^{Q}=0$ ).

The optimum feedback coefficients are given by

$$
B_{k, p}^{\left(p^{\prime}\right)}=\sum_{l^{\prime}=0}^{K-1} F_{k+l^{\prime} M, p} H_{k+l^{\prime} M, p^{\prime}}-\delta_{p, p^{\prime}},
$$

$p^{\prime}=1,2, \ldots, P,\left(\delta_{p, p^{\prime}}=1\right.$ if $p=p^{\prime}$ and 0 otherwise $)$.

${ }^{2}$ It is assumed that the transmitted symbols are selected from a QPSK (Quaternary Phase Shift Keying) constellation under a Gray mapping rule (the generalization to other cases is straightforward).

\section{Performance Results}

In this section, we present a set of performance results concerning the proposed receivers for CCI suppression. We consider the uplink transmission within a CP-assisted SCFDE scheme where $P$ users share the same bandwidth. Each user transmits $M$ data symbols sharing a total bandwidth $K$ times higher than the symbol rate, corresponding to blocks of $N=K M=256$ samples. The duration of the useful part of the data blocks is $4 \mu \mathrm{s}$ and the CP has duration $1.1 \mu \mathrm{s}$. The data symbols are selected from a QPSK constellations under a Gray mapping rule. The radio channel is characterized by the power delay profile type $\mathrm{C}$ for HIPERLAN/2 (HIgh PERformance Local Area Network) [14], with uncorrelated Rayleigh fading on the different paths. A linear power amplification is considered at each transmitter and perfect synchronization and channel estimation are assumed at the receiver. For the sake of comparisons, we included the matched filter bound (MFB) performance.

Let us first consider uncoded performances and a scenario with $P=1,2$ or 3 users employing a transmitter pulse shaping filter with minimum shift-keying (MSK) shape PSD. In this case, the bandwidth of main lobe of the spectrum is three times larger than the symbol rate (i.e., three times the minimum Nyquist bandwidth), not to mention the residual side lobes. Fig. 3 presents the average uncoded BER performance, averaged over all users, for iterations 1, 2 and 4 (naturally, the first iteration corresponds to a linear FDE receiver). Clearly, the iterative receiver allows good CCI suppression with significant performance improvement relatively to the conventional linear FDE after just 3 or 4 iterations. These gains are especially higher when the number of users and the corresponding CCI levels are higher.

Fig. 4 shows the average uncoded BER performance, but corresponding to a scenario with $P=1$ or 2 users, each one employing square-root raised-cosine pulses (i.e., their

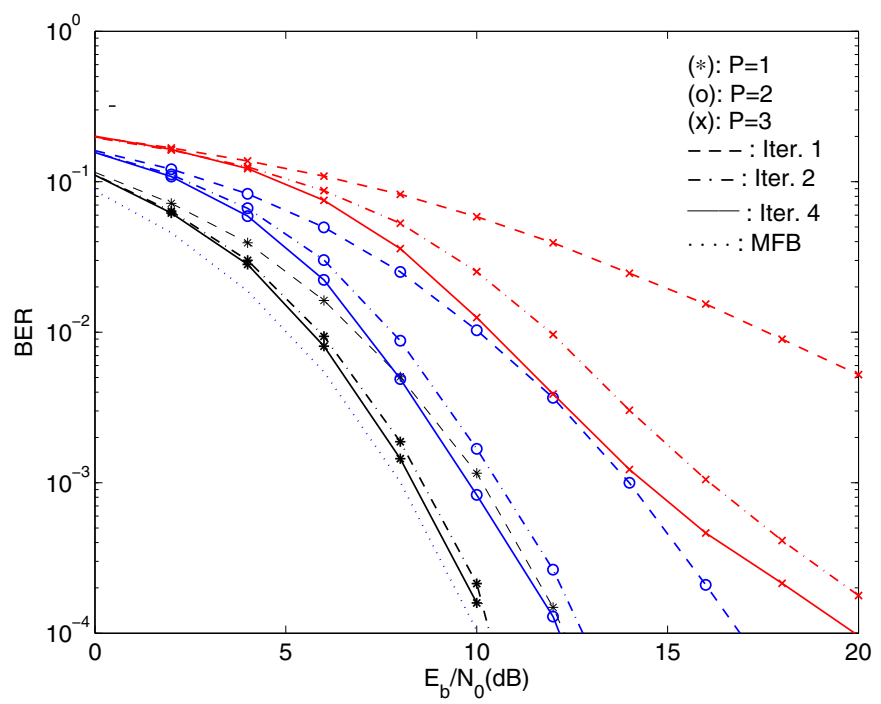

Fig. 3. Average uncoded BER performance for iterations 1, 2 and 4, and MSK pulses. 


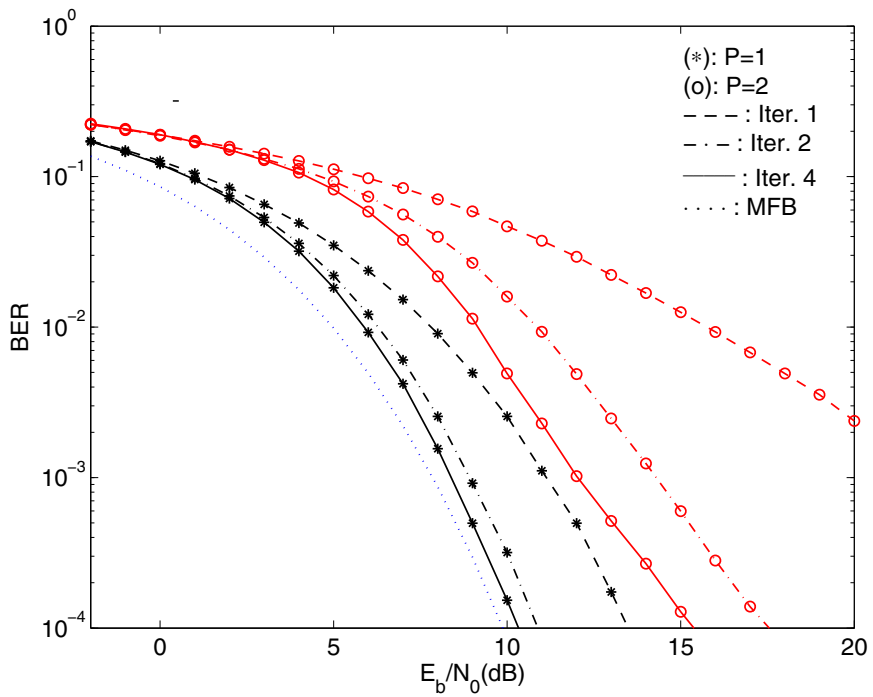

Fig. 4. As in Fig 3, but for square-root raised-cosine pulses with roll-off factor 1 .

PSD has a raised-cosine shape). The roll-off factor is 1, which means that the bandwidth is twice the symbol rate (i.e., twice the minimum Nyquist bandwidth). As in Fig. 3 , it is possible to have two users sharing the band, with only moderate performance degradation when we employ our iterative receiver.

Let us consider now a scenario where the power assigned to different users have different average powers at the receiver. We consider $P=3$ users, denoted by $C_{L}, C_{M}$ and $C_{H}$. The performance results presented in Fig. 5 concerns the case where the average power of $C_{H}$ user is $6 \mathrm{~dB}$ and $12 \mathrm{~dB}$ above the average power of $C_{M}$ user and $C_{L}$ user, respectively, and MSK pulses. Fig. 6 concerns the case with $P=2$ users, where the average power of $C_{H}$ user is $6 \mathrm{~dB}$ above the average power of $C_{L}$ user and square-root raisedcosine pulses with roll-off factor 1 . Clearly, the $C_{L}$ users face stronger CCI levels. Once again, the proposed iterative receiver allows significant performance gain, specially for the low power users. The higher BER of low-power users preclude an appropriate interference cancelation when detecting highpower users.

Let us consider now the impact of channel coding. We consider the well-known rate-1/2, 64-state convolutional code with generators $1+D^{2}+D^{3}+D^{5}+D^{6}$ and $1+D+D^{2}+D^{3}+D^{6}$. The coded bits are interleaved before being mapped into QPSK symbols. To improve performances we consider a "turboreceiver" that, as turbo equalizers, employs the "soft-decision" from the SISO (Soft-in, Soft-out) channel decoder outputs instead of the "soft-decision" from the multiuser detector. The SISO block, that can be implemented as defined in [15], provides the LLRs of both the "information bits" and the "coded bits". The input of the SISO block are LLRs of the "coded bits" at the multiuser detector. Figs. 7 shows the average coded BER performance for iterations 1 and 4 , for the same scenario of Fig. 3. As expected, the channel coding

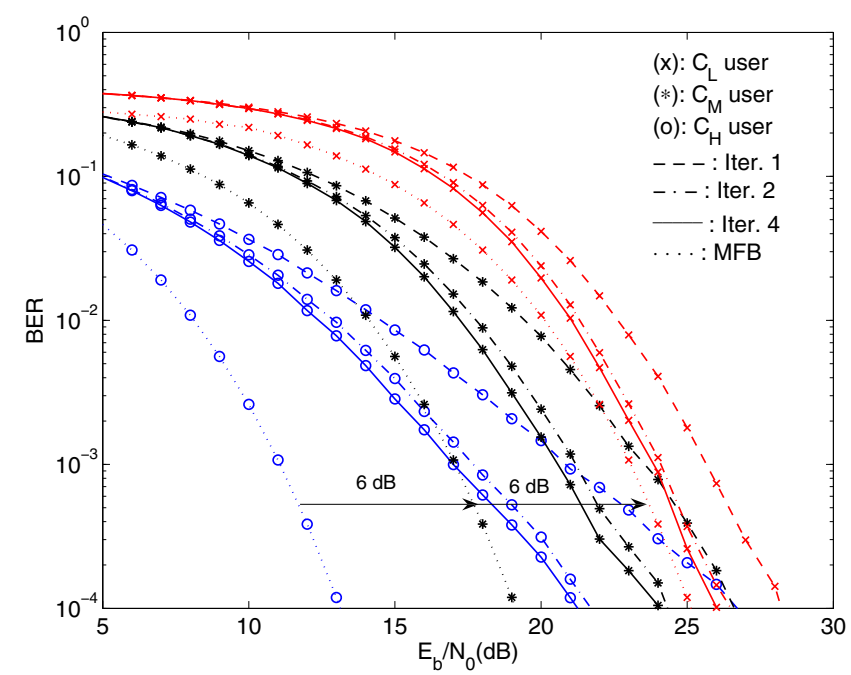

Fig. 5. BER performance for iterations 1,2 and 4 as a function of the $E_{b} / N_{0}$ of $C_{H}$ user (average power of $C_{H}$ user $6 \mathrm{~dB}$ and $12 \mathrm{~dB}$ above the average power of $C_{M}$ user and $C_{L}$ user, respectively), and MSK pulses.

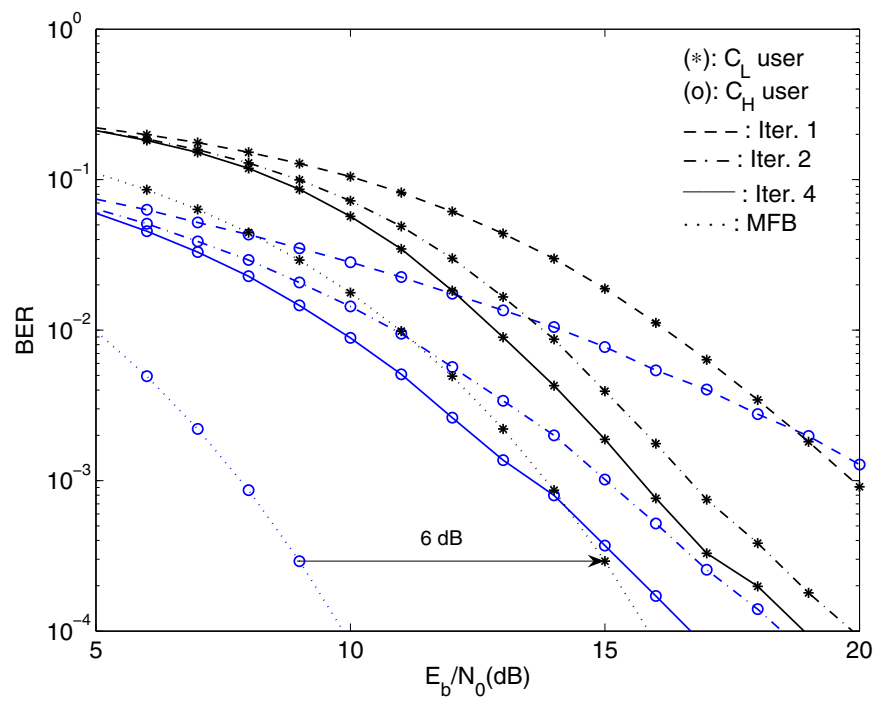

Fig. 6. BER performance for iterations 1,2 and 4 as a function of the $E_{b} / N_{0}$ of $C_{H}$ user (average power of $C_{H}$ user $6 \mathrm{~dB}$ above the average power of $C_{L}$ user), and square-root raised-cosine pulses with roll-off factor 1 .

leads to significant performance improvements.

\section{CONCLUSIONS}

In this paper we considered SC-FDE schemes where the transmission bandwidth is above the symbol rate and several channels share the same bandwidth. To cope with the high CCI levels, we proposed iterative FDE receivers where we jointly detect all users sharing the same channel.

Our performance results showed that we can have excellent performances, even with several users sharing the same channel. In fact, in some cases we can have the maximum theoretical spectral efficiency even with signals that have bandwidth substantially above the symbol rate. 


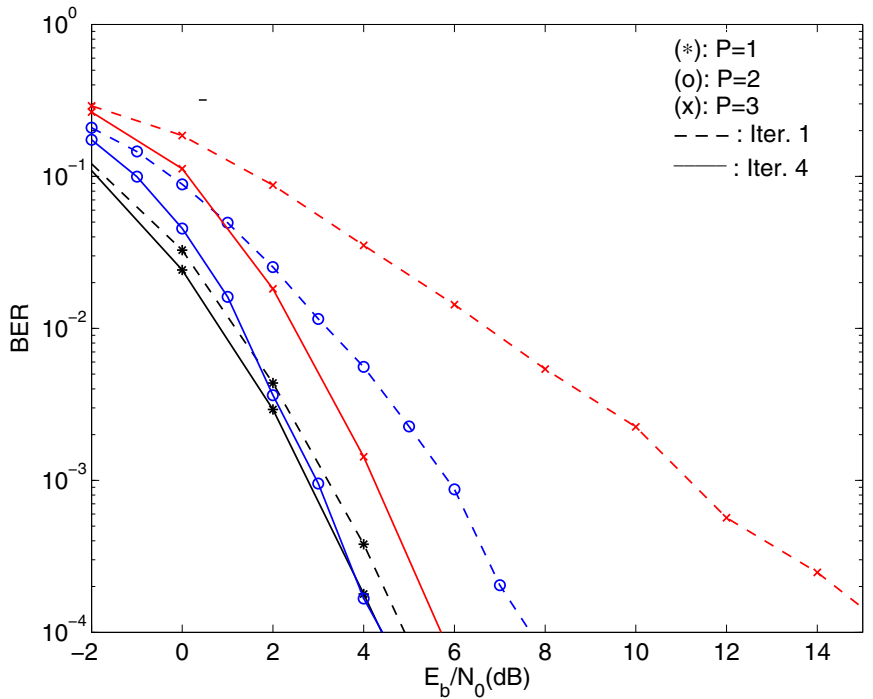

Fig. 7. Average coded BER performances for iterations 1 and 4 for the same scenario of Fig. 3.

\section{REFERENCES}

[1] H. Sari, G. Karam and I. Jeanclaude, "An Analysis of Orthogonal Frequency-division Multiplexing for Mobile Radio Applications", In Proc. IEEE Vehic. Tech. Conf., VTC'94, pp. 1635-1639, Stockholm, June 1994.

[2] A. Gusmão, R. Dinis, J. Conceição, and N. Esteves, "Comparison of Two Modulation Choices for Broadband Wireless Communications", IEEE VTC'OO (Spring), Tokyo, Japan, May 2000.

[3] D. Falconer, S. Ariyavisitakul, A. Benyamin-Seeyar and B. Eidson, "Frequency Domain Equalization for Single-Carrier Broadband Wireless Systems", IEEE Comm. Mag., Vol. 4, No. 4, pp. 58-66, April 2002.

[4] W. Gardner, "Introduction to Random Processes with Applications to Signals and Systems", McGraw-Hill, 1990.

[5] W. Gardner, "Exploitation of Spectral Redundancy in Cyclostationary Signals", IEEE Comm. Mag., Vol. 8, No. 2, pp. 14-36, Apr. 1991.

[6] B. Petersen and D. Falconer, "Minimum Mean Square Equalization in Cyclostationary and Stationary Interference-Analysis and Subscriber Line Calculations", IEEE Journal on Sel. Areas in Comm., Vol. 9, No. 56, pp. 931-940, Aug. 1991.

[7] B. Petersen and D. Falconer, "Suppression of Adjacent Channel, Co-channel, and Intersymbol Interference by Equalizers and Linear Combiner", IEEE Trans. on Comm., Vol. 42, No. 12, pp. 3109-3118, Dec. 1994.

[8] R. Dinis, D. Falconer and B. Ng, "Iterative Frequency-Domain Equalizers for Adjacent Channel Interference Suppression", IEEE GLOBECOM'05, St. Louis, USA, Nov.-Dec. 2005.

[9] M. Luzio, R. Dinis and P. Carvalho, "Frequency-Domain Parallel Multiuser Detection for Quasi-Constant Envelope OQPSK Schemes with High Spectral Efficiency", IEEE ICSPCS, Omaha, USA, Sep. 2009.

[10] S. Okuyama, k. Takeda and F. Adachi, "Frequancy-Domain Iterative MUI Cancellation for Uplink SC-FDMA Using FrequencyDomain Filtering", IEEE VTC'10-Fall, pp. 1-5, Ottawa, Sep. 2010.

[11] N. Benvenuto and S. Tomasin, "Block Iterative DFE for Single Carrier Modulation”, IEE Elec. Let., Vol. 39, No. 19, pp. 11441145, Sep. 2002.

[12] N. Benvenuto, R. Dinis, D. Falconer and S. Tomasin, "Sin- gle Carrier Modulation with Non Linear Frequency Domain Equalization: An Idea Whose Time Has Come - Again", IEEE Proceedings, Vol. 98, No. 1, pp. 69-96, Jan. 2010.

[13] P. Silva and R. Dinis, "Turbo Detection for the Uplink of CPAssisted DS-CDMA Signals", IEEE PACRIM'07, Victoria, Aug 2007.

[14] ETSI, "Channel models for HIPERLAN/2 in Different Indoor Scenarios", ETSI EP BRAN 3ERI085B, pp. 1-8, March 1998.

[15] B.Vucetic and J.Yuan, Turbo Codes: Principles and Applications, Kluwer Academic Publ., 2002. 